Proceedings of the Edinburgh Mathematical Society (2003) 46, 379-382 (C)

DOI:10.1017/S0013091500001085 Printed in the United Kingdom

\title{
EPIMORPHISMS ONTO DERIVATION ALGEBRAS
}

\author{
A. R. VILLENA \\ Departamento de Análisis Matemático, Facultad de Ciencias, \\ Universidad de Granada, 18071 Granada, Spain (avillena@ugr.es)
}

(Received 2 November 2000)

\begin{abstract}
We prove that the separating space of an epimorphism from a Lie-Banach algebra onto the (continuous) derivation algebra $\mathfrak{D e r}(A)$ of a Banach algebra $A$ consists of derivations which map into the radical of $A$.
\end{abstract}

Keywords: derivations; Lie-Banach algebras; Lie epimorphisms

2000 Mathematics subject classification: Primary 46H40; 46H70

Secondary 47B47; 47B48

For every Banach algebra $A$ let $\mathfrak{D e r}(A)$ denote the linear space of all continuous derivations on $A$. It is easily checked that $\operatorname{Der}(A)$ is a closed Lie subalgebra of the Banach algebra $L(A)$ of all continuous linear operators on $A$. In [5] it was proved that in the case where $A$ is a $C^{*}$-algebra, the topology on $\mathfrak{D e r}(A)$ inherited from $L(A)$ is the unique Banach space topology which makes the Lie bracket $[\cdot, \cdot]: \mathfrak{D e r}(A) \times \mathfrak{D e r}(A) \rightarrow \operatorname{Der}(A)$ continuous. We show that the preceding property is still true even in the case where $A$ is any semisimple Banach algebra. This clearly follows from the following theorem, which will be proved in this paper.

Theorem 1. Let $A$ be a Banach algebra, let $\mathfrak{L}$ be a Lie-Banach algebra, and let $\Phi$ be an epimorphism from $\mathfrak{L}$ onto $\mathfrak{D e r}(A)$. Then $D(A) \subset \operatorname{Rad}(A)$ for each $D$ in the separating space of $\Phi$. Accordingly, $\Phi$ is continuous in the case where $A$ is semisimple.

By a Lie-Banach algebra we mean a complex Lie algebra whose underlying linear space is a Banach space such that the product $[\cdot, \cdot]: \mathfrak{L} \times \mathfrak{L} \rightarrow \mathfrak{L}$ is continuous. For every $x \in \mathfrak{L}$ let $\operatorname{ad}_{\mathfrak{L}}(x)$ denote the continuous linear operator $\operatorname{ad}_{\mathfrak{L}}(x)(y)=[x, y]$ from $\mathfrak{L}$ into itself. It is easily seen that $\left[D, \operatorname{ad}_{\mathfrak{L}}(x)\right]=\operatorname{ad}_{\mathfrak{L}}(D(x))$ for each $x \in \mathfrak{L}$ and each derivation $D$ on $\mathfrak{L}$. An ideal $I$ of $\mathfrak{L}$ is said to be abelian if $[I, I]=0$, and $\mathfrak{L}$ is said to be semisimple if it has no non-zero abelian ideal. The standard examples of Lie-Banach algebras are the closed Lie subalgebras of a Banach algebra $A$, such as $A$ itself, the skew elements $K_{A}$ of an $A$ in the case where $A$ is endowed with a continuous linear involution, and the derivation algebra $\mathfrak{D e r}(A)$ of $A$. The continuity of Lie epimorphisms onto $A$ was studied in $[2]$ and the continuity of isomorphisms onto $K_{A}$ was studied in [3]. Throughout this paper, $\mathfrak{L}$ stands for a Lie-Banach algebra, $A$ stands for a complex Banach algebra with 
radical $\operatorname{Rad}(A)$, and $\Phi$ stands for an epimorphism from $\mathfrak{L}$ onto $\mathfrak{D e r}(A)$. We measure the continuity of $\Phi$ by considering its separating space $\mathfrak{S}(\Phi)$. The separating space of a linear map $T: X \rightarrow Y$, where $X$ and $Y$ are Banach spaces, is defined as

$$
\mathfrak{S}(T)=\left\{y \in Y: \text { there exists } x_{n} \rightarrow 0 \text { in } X \text { with } T x_{n} \rightarrow y\right\} .
$$

The closed graph theorem shows that $T$ is continuous if and only if $\mathfrak{S}(T)=0$. For every Banach space $X$ let $L(X)$ denote the Banach algebra of all continuous linear operators from $X$ into itself.

For every $b$ in a Banach algebra $B$ let $r(b, B)$ denote the spectral radius of $b$. The spectral arguments were brought into automatic continuity theory by Aupetit $[\mathbf{1}]$ and they have proved to be very useful for studying the automatic continuity of epimorphisms onto Banach algebras. The following result illustrates this technique and it can be easily derived from $[\mathbf{1}, \mathbf{4}]$.

Lemma 2. Let $X$ be a Banach space, let $B$ be a Banach algebra, and let $\phi$ be a linear map from $X$ into $B$. Suppose that there exists $M>0$ such that $r(\phi(x), B) \leqslant M\|x\|$ for each $x \in X$. Then

$$
r(\phi(x), B)^{2} \leqslant M\|x\| \operatorname{dist}(\phi(x), \mathfrak{S}(\phi))
$$

for each $x \in X$. Accordingly, $\mathfrak{S}(\phi) \cap \phi(X)$ consists of quasinilpotent elements.

A second ingredient in the proof of our theorem is the following result.

Lemma 3. Let $a \in A$. Then

$$
r\left(\operatorname{ad}_{\mathfrak{D e r}(A)}\left(\operatorname{ad}_{A}(x)\right), L(\mathfrak{D e r}(A))\right)=r\left(\operatorname{ad}_{A}(a), L(A)\right) .
$$

Proof. An easy computation shows that

$$
\left(\operatorname{ad}_{\mathfrak{D e r}(A)}\left(\operatorname{ad}_{A}(a)\right)\right)^{n+1}(D)=-\operatorname{ad}_{A}\left(\left(\operatorname{ad}_{A}(a)\right)^{n}(D(a))\right)
$$

for all $D \in \mathfrak{D e r}(A)$ and $n \in \mathbb{N}$.

From this we conclude that

$$
\begin{aligned}
\left\|\left(\operatorname{ad}_{\mathfrak{D e r}(A)}\left(\operatorname{ad}_{A}(a)\right)\right)^{n+1}(D)\right\| & \leqslant 2\left\|\left(\operatorname{ad}_{A}(a)\right)^{n}(D(a))\right\| \\
& \leqslant 2\left\|\left(\operatorname{ad}_{A}(a)\right)^{n}\right\|\|D\|\|a\|,
\end{aligned}
$$

and, hence, that

$$
\left\|\left(\operatorname{ad}_{\mathfrak{D e r}(A)}\left(\operatorname{ad}_{A}(a)\right)\right)^{n+1}\right\| \leqslant 2\left\|\left(\operatorname{ad}_{A}(a)\right)^{n}\right\|\|a\|,
$$

and finally that

$$
r\left(\operatorname{ad}_{\mathfrak{D e r}(A)}\left(\operatorname{ad}_{A}(a)\right), L(\mathfrak{D e r}(A))\right) \leqslant r\left(\operatorname{ad}_{A}(a), L(A)\right) .
$$

For every $b \in A$, from the first equality we deduce that

$$
\left(\operatorname{ad}_{\mathfrak{D e r}(A)}\left(\operatorname{ad}_{A}(a)\right)\right)^{n+1}\left(\operatorname{ad}_{A}(b)\right)(a)=-\left(\operatorname{ad}_{A}(a)\right)^{n+2}(b) .
$$


Hence

$$
\left\|\left(\operatorname{ad}_{A}(a)\right)^{n+2}(b)\right\| \leqslant\left\|\left(\operatorname{ad}_{\mathfrak{D e r}(A)}\left(\operatorname{ad}_{A}(a)\right)\right)^{n+1}\right\| 2\|b\|\|a\| .
$$

From this we finally see that

$$
r\left(\operatorname{ad}_{A}(a), L(A)\right) \leqslant r\left(\operatorname{ad}_{\mathfrak{D e r}(A)}\left(\operatorname{ad}_{A}(a)\right), L(\mathfrak{D e r}(A))\right) .
$$

Lemma 4. Let $I$ be an ideal of $\mathfrak{D e r}(A)$ such that $\operatorname{ad}_{\mathfrak{D e r}(A)}(D)$ is quasinilpotent for each $D \in I$. Then $D(A) \subset \operatorname{Rad}(A)$ for each $D \in I$.

Proof. Set $D \in I$. For all $a, b \in A$ we have

$$
\operatorname{ad}_{A}([D(a), b])=\left[\left[D, \operatorname{ad}_{A}(a)\right], \operatorname{ad}_{A}(b)\right] \in I .
$$

Thus $\operatorname{ad}_{\mathfrak{D e r}(A)}\left(\operatorname{ad}_{A}([D(a), b])\right)$ is quasinilpotent and we can see that $\operatorname{ad}_{A}([D(a), b])$ is quasinilpotent from Lemma 3.

From [2, Proposition] it may be concluded that $[D(A), A] \subset \operatorname{Rad}(A)$ and therefore $[6]$ now gives $D(A) \subset \operatorname{Rad}(A)$.

Corollary 5. Let $A$ be a Banach algebra and let $I$ be an abelian ideal of $\mathfrak{D e r}(A)$. Then $D(A) \subset \operatorname{Rad}(A)$ for each $D \in I$. Accordingly, $\mathfrak{D e r}(A)$ is a semisimple Lie algebra in the case where $A$ is semisimple.

Proof. On account of the above lemma, it suffices to show that $\operatorname{ad}_{\mathfrak{D e r}(A)}(D)$ is quasinilpotent for each $D \in \mathfrak{D e r}(A)$. Set $D \in I$. For every $D^{\prime} \in I$ we have $\left[D, D^{\prime}\right] \in I$ and so $\left[D,\left[D, D^{\prime}\right]\right]=0$. Therefore, $\left(\operatorname{ad}_{\mathfrak{D e r}(A)}(D)\right)^{2}=0$, which shows that $\operatorname{ad}_{\mathfrak{D e r}(A)}(D)$ is nilpotent.

Proof of Theorem 1. It follows immediately that $\mathfrak{S}(\Phi)$ is an ideal of $\mathfrak{D e r}(A)$.

On the other hand, let $\phi: \mathfrak{L} \rightarrow L(\mathfrak{D e r}(A))$ be the linear map given by $\phi(x)=$ $\operatorname{ad}_{\mathfrak{D e r}(A)}(\Phi(x))$ for all $x \in \mathfrak{L}$. For every $x \in \mathfrak{L}$, we have $\Phi \circ \operatorname{ad}_{\mathfrak{L}}(x)=\operatorname{ad}_{\mathfrak{D e r}(A)}(\Phi(x)) \circ \Phi$. Since $\Phi$ is surjective, a standard result from spectral theory shows that

$$
r\left(\operatorname{ad}_{\mathfrak{D e r}(A)}(\Phi(x)), L(\mathfrak{D e r}(A))\right) \leqslant r\left(\operatorname{ad}_{\mathfrak{L}}(x), L(\mathfrak{L})\right) \leqslant\left\|\operatorname{ad}_{\mathfrak{L}}(x)\right\| \leqslant M\|x\|,
$$

where we write $M$ for the norm of the product of $\mathfrak{L}$. Consequently, $\phi$ satisfies the requirement in Lemma 2. Set $D \in \mathfrak{S}(\Phi)$. There is $x \in \mathfrak{L}$ such that $\Phi(x)=D$ and therefore $\phi(x)=\operatorname{ad}_{\mathfrak{D e r}(A)}(D)$. From Lemma 2 it follows that $\operatorname{ad}_{\mathfrak{D e r}(A)}(D)$ is quasinilpotent and Lemma 4 now shows that $D(A) \subset \operatorname{Rad}(A)$.

Corollary 6. Let $A$ be a semisimple Banach algebra. Then $\mathfrak{D e r}(A)$ carries a unique Lie-Banach algebra topology.

According to Theorem 1 and Corollary 5 , it is to be expected that every derivation on $\operatorname{Der}(A)$ is continuous in the case where $A$ is semisimple, but we have not been able to prove this. The main difficulty in investigating this problem is that the spectral techniques have not proved to be too useful when dealing with derivations. 
Question. Let $A$ be a semisimple Banach algebra. Is every derivation $\Delta: \mathfrak{D e r}(A) \rightarrow$ $\mathfrak{D e r}(A)$ automatically continuous?

Acknowledgements. The author is supported by DGICYT grant PB98-1358.

\section{References}

1. B. Aupetit, The uniqueness of the complete norm topology in Banach algebras and Banach Jordan algebras, J. Funct. Analysis 47 (1982), 1-6.

2. B. Aupetit and M. Mathieu, The continuity of Lie homomorphisms, Studia Math. 138 (2000), 193-199.

3. M. I. Berenguer And A. R. Villena, Continuity of Lie mappings of the skew elements of Banach algebras with involution, Proc. Am. Math. Soc. 126 (1998), 2717-2720.

4. T. J. RANSFORD, A short proof of Johnson's uniqueness-of-norm theorem, Bull. Lond. Math. Soc. 21 (1989), 487-488.

5. A. Rodríguez, The uniqueness of the complete norm topology in complete normed nonassociative algebras, J. Funct. Analysis 60 (1985), 1-15.

6. B. Yood, Continuous homomorphisms and derivations on Banach algebras, in Contemporary mathematics, vol. 32, pp. 279-284 (American Mathematical Society, Providence, RI, 1984). 